\title{
Erratum to: A note on the motion representation and configuration update in time stepping schemes for the constrained rigid body
}

\section{Andreas Müller ${ }^{1}$}

\section{Erratum to: BIT Numer Math DOI 10.1007/s10543-015-0580-y}

Due to typesetting errors, some corrections marked in the proof were not carried out.

1. The line after Eq. (6): The argument in $\exp \widehat{\xi}$ should be in boldface.

2. The second line after Eq. (8): Superscripts in the equation should not be in boldface: $\mathbf{V}^{\mathrm{m}}=\left(\boldsymbol{\omega}^{\mathrm{b}}, \dot{\mathbf{r}}\right)$.

3. The third line after (8): The vector should be in boldface: $\omega^{\mathrm{b}}$.

4. The fourth line after Eq. (11): The superscripts should not be in boldface: $\left(\boldsymbol{\Theta}_{0} \boldsymbol{\omega}^{\mathrm{b}}, m \dot{\mathbf{r}}\right) \in s o^{*}(3) \times \mathbb{R}^{3}$.

5. The Eq. (12) should be

$$
\begin{aligned}
\boldsymbol{\Theta}_{0} \dot{\boldsymbol{\omega}}^{\mathrm{b}}+\boldsymbol{\omega}^{\mathrm{b}} \times \boldsymbol{\Theta}_{0} \boldsymbol{\omega}^{\mathrm{b}} & =\mathbf{0} \\
m \ddot{\mathbf{r}} & =\mathbf{0} .
\end{aligned}
$$

6. The Eq. (17) should be

$$
\begin{aligned}
\boldsymbol{\Theta}_{0} \dot{\boldsymbol{\omega}}^{\mathrm{b}}+\boldsymbol{\omega}^{\mathrm{b}} \times \boldsymbol{\Theta}_{0} \boldsymbol{\omega}^{\mathrm{b}}=\mathbf{0} \\
m \dot{\mathbf{v}}^{\mathrm{b}}+m \boldsymbol{\omega}^{\mathrm{b}} \times \mathbf{v}^{\mathrm{b}}=\mathbf{0} .
\end{aligned}
$$

7. The first line after Eq. (18): The equation should be $\mathbf{J}(C) \dot{\mathbf{V}}=\eta(C, \mathbf{V})$.

The online version of the original article can be found under doi:10.1007/s10543-015-0580-y.

$凶 \quad$ Andreas Müller

a.mueller@jku.at

1 Johannes Kepler Universität Linz, Linz, Austria 
8. The Eq. (23) should read

$$
\mathbf{J} \dot{\mathbf{V}}^{\mathrm{b}}=\mathbf{R}(\widehat{\boldsymbol{\omega}} \widehat{\omega} \mathbf{p}+\widehat{\boldsymbol{\omega}} \mathbf{v}), \text { with } \mathbf{J}=(\mathbf{R} \widehat{\mathbf{p}}-\mathbf{R})
$$

9. The second line of the second paragraph in Sect. 5.3: It should be $\mathbf{p} \times \omega_{0}^{\mathrm{b}}+(0,1,0)$ $\mathrm{m} / \mathrm{s}$. 